\title{
Measurements of Preformed Plasma Generation and Its Suppression Inside a Cone in a Cone-in-Shell Target for Fast Ignition
}

\author{
Shoji HATTORI, Keisuke SHIGEMORI, Yoichiro HIRONAKA, Takumi YAMAMOTO, \\ Hiroki KATO, Shinsuke FUJIOKA, Hiroyuki SHIRAGA and Hiroshi AZECHI \\ Institute of Laser Engineering, Osaka University, 2-6 Yamada-Oka, Suita, Osaka 565-0871, Japan
}

(Received 12 May 2015 / Accepted 30 July 2015)

\begin{abstract}
We have investigated preformed plasma generation inside a cone on cone-in-shell targets for fast ignition scheme. We focused on the study of preformed plasma generation via irradiation by an implosion laser. In the GEKKO-XII laser system (Institute of Laser Engineering, Osaka University), a large fraction of laser energy is frequency-doubled to a $527 \mu \mathrm{m}$ wavelength light with KDP crystals, but unconverted fundamental light $(\lambda=$ $1.053 \mu \mathrm{m})$ is ultimately a possible heating source for the target. We measured the temperature at the inside tip of the cone in several experimental conditions to verify the effect of unconverted light. The experimental results indicated that the direct irradiation inside the cone with the unconverted fraction is the primary source of preheating effects. The results also suggest that preheating is suppressed by "long" cone targets whose sizes are typically larger than the focal points of incident unconverted light.
\end{abstract}

(C) 2015 The Japan Society of Plasma Science and Nuclear Fusion Research

Keywords: fast ignition, cone-in-shell target, unconverted light, streaked optical pyrometer

DOI: $10.1585 / \mathrm{pfr} .10 .1404076$

\section{Introduction}

On the fast ignition scheme of inertial confinement fusion targets, imploded high-density fuel is heated by igniting an ultra-intense laser [1,2]. For a typical fast ignition scheme, the igniting laser is irradiated into the "cone" of a cone-in-shell target at the maximum compression timing. High-energy electrons and ions are generated by the irradiation with the ultra-intense laser, which is the source of heating. There is certain optimum energy of electrons for heating compressed fuel because high-energy electrons penetrate fuel plasma, whereas low-energy electrons do not reach the fuel because of absorption by the cone wall made of high-atomic-number material.

Previous experimental investigations suggest that a large fraction of high-energy electrons is the main factor behind the degree of efficient heating [3]. The physical mechanism behind the generation of high-energy electrons is supposedly due to the engagement of preformed plasma, which is generated on the target surface prior to irradiation by the ultra-intense laser. Electrons are typically accelerated (to high-energy levels) in such plasmas between cutoff surfaces and target surfaces [4]. The most probable source of preformed plasma is likely associated with the prepulse and/or pedestal of an igniting short-pulse laser. In recent experiments at our GEKKO-XII laser facility, it was found that the tip of the cone is heated prior to the maximum fuel compression level, even without igniting

author'se-mail: shige@ile.osaka-u.ac.jp the short-pulse laser [5]. This fact indicates that the existence of preformed plasma due to the laser itself is also a very important factor.

In this study, experiments regarding the generation of preformed plasma inside a cone in a cone-in-shell target for fast ignition are discussed. We performed optical diagnostic measurements of self-emissions at inside tips of the subject cones. Datasets were obtained by changing laser conditions and target parameters to verify (and ultimately eliminate) the source(s) of preformed plasma inside the cones.

\section{Preformed Plasma Generation in- side a Cone by Laser for Implosion}

The mechanism of the preformed plasma generation inside a cone in the cone-in-shell target is due to several factors. Figure 1 shows possible sources of preheating at the tip of the cone. When the fuel shell target is irradiated with the laser for implosion, the shell is accelerated by ablation pressure. On irradiation, high-energy electrons and $\mathrm{x}$-rays may then penetrate into the shell and reach the tip of the cone. The ablation plasma itself may be a source of heating when the blow-off plasma moves around and inside the cone. The wall of the cone may also be heated by the imploding shell plasma via thermal heat conduction.

Another probable source is the unconverted light of the irradiation laser. At the aforementioned GEKKO-XII laser facility [6], the fundamental light $(\lambda=1.053 \mu \mathrm{m})$ 


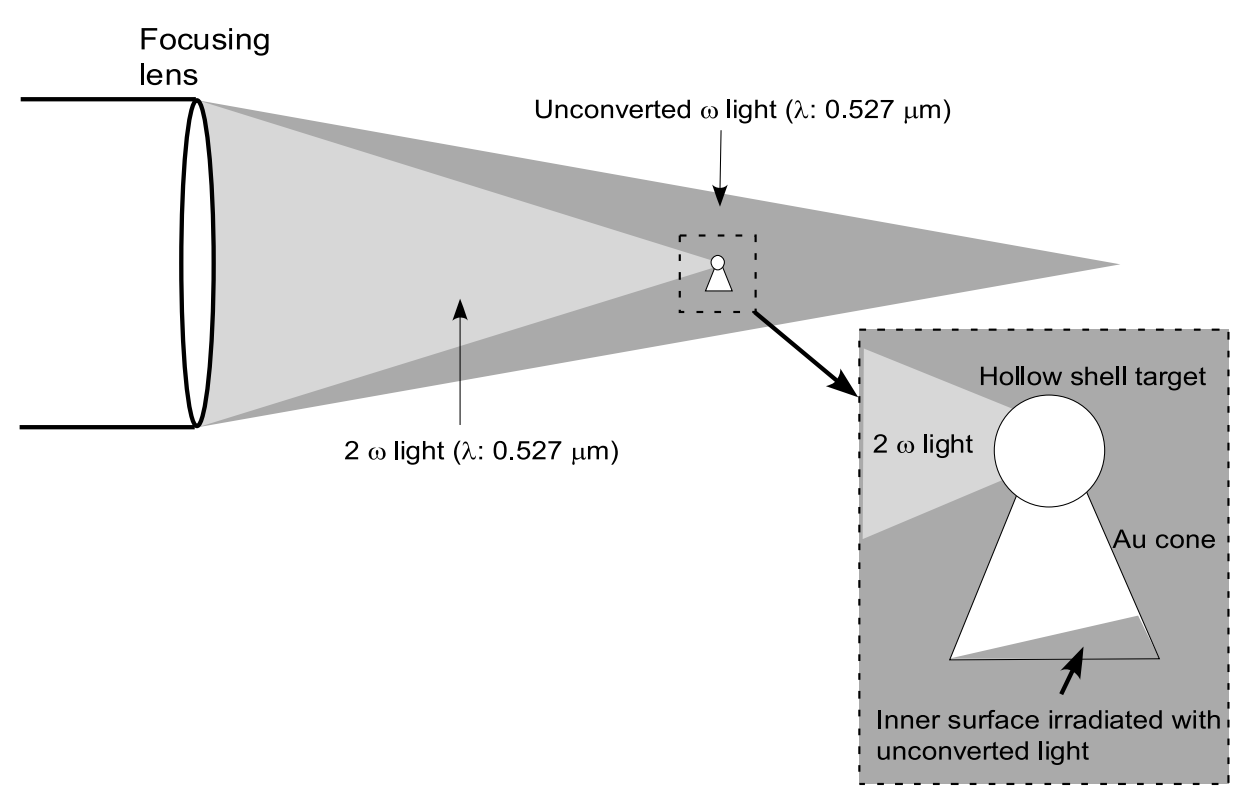

Fig. 1 Irradiation configuration of cone-in-shell target at GEKKO-XII facility.

is converted to a second-harmonic wavelength light $(\lambda=$ $0.527 \mu \mathrm{m})$ by KDP crystals. The second-harmonic light is focused on the target, whereas the fundamental light is also focused on the target but with a larger spot size because of the chromatic aberration of the focusing lens. A schematic of the irradiation configuration of the GEKKOXII laser system is shown in Fig. 1. Although the spot size for unconverted light is relatively large $(\sim 8 \mathrm{~mm})$, the effective intensity on the target is around $10^{12} \mathrm{~W} / \mathrm{cm}^{2}$, which is not negligible for plasma generation. Unconverted light irradiates outside and inside the cone.

Although the heating sources are from inside and outside the cone, the heating from the outside would be negligible because the opacity of the $7-\mu \mathrm{m}$-thick gold cone is sufficiently large to shield typical heating from the outside source described above. Thus, focus was ultimately placed on the heating source inside the cone, which is essentially the unconverted light of the implosion laser. The effect of such unconverted light has also been an important problem at the OMEGA laser facility, as per research conducted in 2009 [7]. However, previous subject experimental results suggest that no preheating inside a cone was observed at the OMEGA facility, where the drive laser does not contain any unconverted light [8].

For standard cone-in-shell target experiments, the inside of a cone is partially irradiated around the edge of the cone by three of nine separate beams. The irradiation of unconverted light at the edge of a cone may create plasma at temperatures associated with energies greater than $200 \mathrm{eV}$, obtained via calculations with the onedimensional simulation code ILESTA-1D [9]. The irradiated surface can potentially emit x-rays and hence immediately heat up the entire inside of the cone.

Because the focused unconverted light has a certain beam size on the target, the effect of unconverted light should theoretically be eliminated by changing the spatial beam pattern or cone size. For the spatial beam pattern, unconverted light does not irradiate the target using a spatial mask, which blocks the central part of the beam in accordance with the cone size. A schematic of the "doughnut" beam is shown in Fig. 2 (a). Another possible approach is to increase the cone size (length), which is illustrated in Fig. 2 (b). The typical length of the cone in previous experiments was $1 \mathrm{~mm}$. When the length of the cone is longer than $5 \mathrm{~mm}$, all unconverted light is irradiated outside the cone. The two separate schemes were tested to verify the effects on the preformed plasma generation.

\section{Experiments}

Experiments were performed at the aforementioned GEKKO-XII laser facility. On the fast ignition experiments, cone-in-shell targets were irradiated with nine out of twelve separate beams of the GEKKO-XII laser. Typical laser energies from the nine beams fell in the range of $2-3 \mathrm{~kJ}$. The wavelength and pulse duration were $527 \mathrm{~nm}$ and $1.3 \mathrm{~ns}$ (Gaussian, full width at half maximum), respectively. To compare the effects on irradiation geometry as per the unconverted light scenario described above, a mask was affixed to the setup, which blocked the central part of the beam (with a diameter of $122 \mathrm{~mm}$ ) for some of the data shots. Masks for three beams were placed just in front of the focusing lens, which created a doughnut-shaped focal pattern for unconverted light on the target chamber center (inner-circle diameter of $3.3 \mathrm{~mm}$ ).

We employed cone-in-shell targets, which consisted of a deuterated plastic (CD) shell $(\sim 500 \mu \mathrm{m}$ diameter and $\sim 7 \mu \mathrm{m}$ thick) and a gold cone, whose opening angle was $45^{\circ}$. The length between the entrance and tip of a standard 
(a)

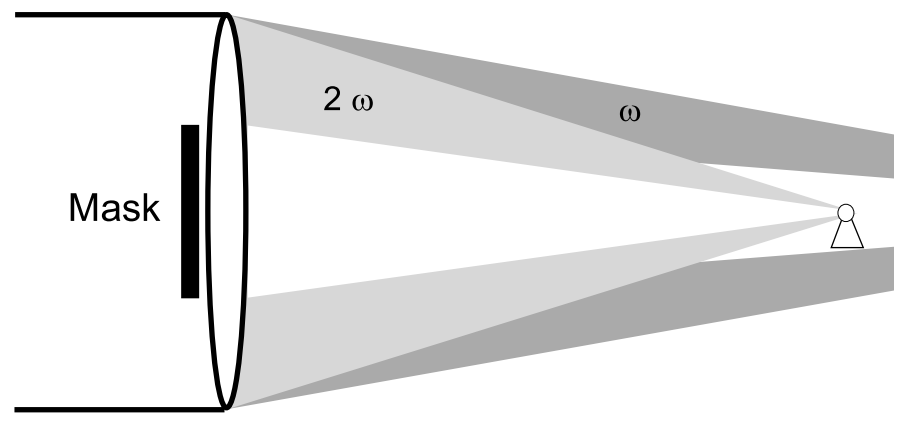

(b)

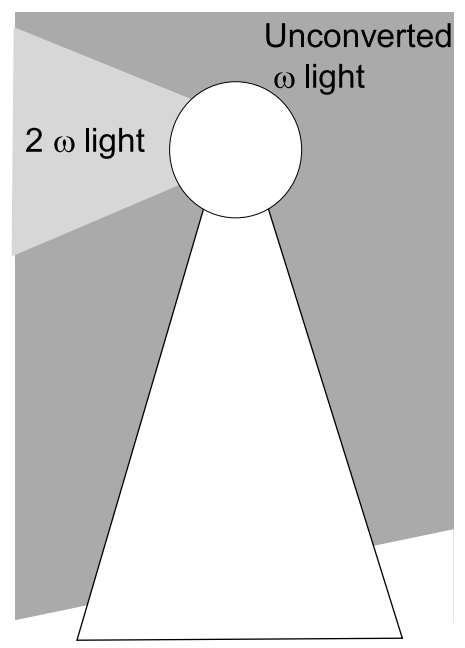

Fig. 2 Irradiation scheme to eliminate unconverted light with (a) doughnut beam shape irradiation with a mask and with (b) long cone target.

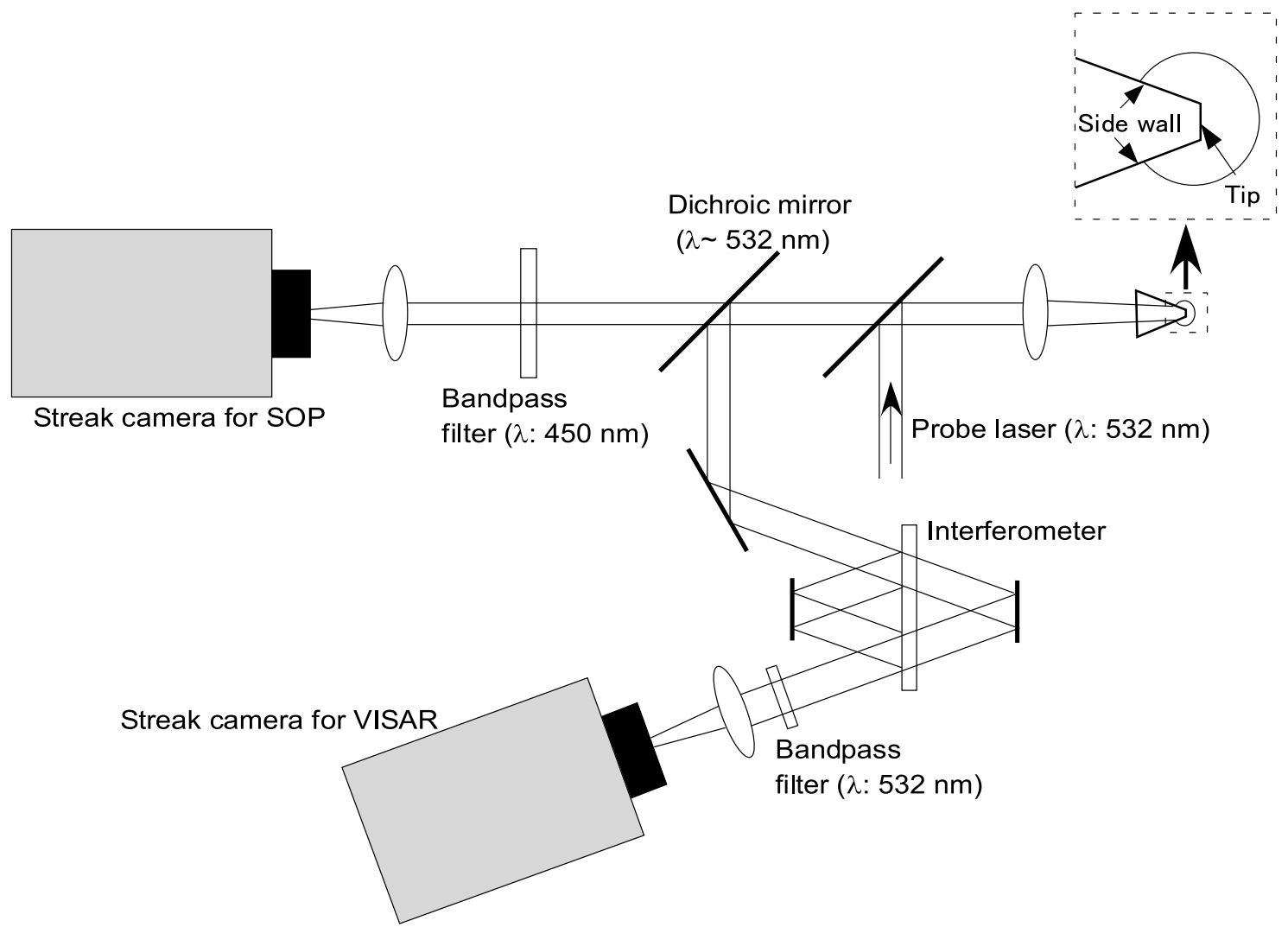

Fig. 3 Arrangements of optical measurements in this experiment.

"short" cone was $1 \mathrm{~mm}$, whereas that of a "long" cone was $5.5 \mathrm{~mm}$. Long cones were utilized in the trial because their greater length aided in eliminating unconverted light irradiation inside the cone. For reflectivity measurements described below, the tip of the cone was flat with a $100 \mu \mathrm{m}$ diameter.

The preformed plasma generation in the cone was observed by optical diagnostics; here, special emphasis was placed on heating parameters at the tip of the cone. A schematic of the optical measurement process is shown in Fig. 3. The tip of the inside of the cone was imaged by lenses of two streak cameras. One of the two cameras was employed to measure self-emissions as a streaked optical pyrometer (SOP) [10]. The spatial and temporal resolutions of the SOP were approximately $15 \mu \mathrm{m}$ and $100 \mathrm{ps}$, respectively. The wavelength intensity was measured at 
(a)

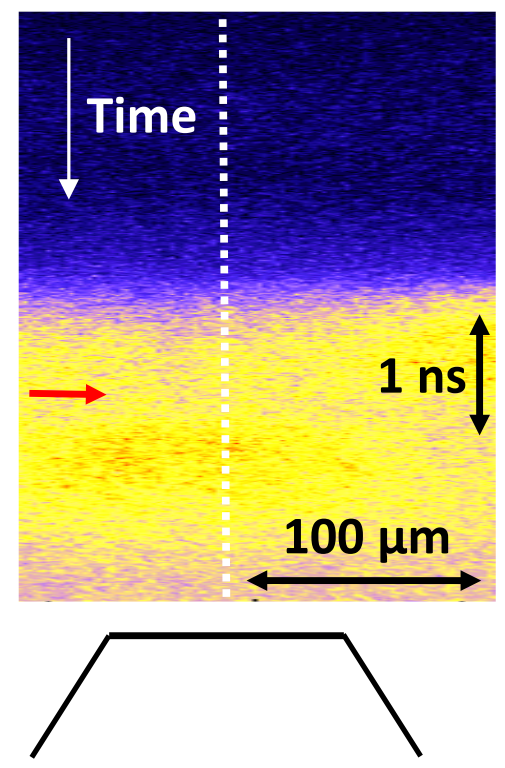

(b)

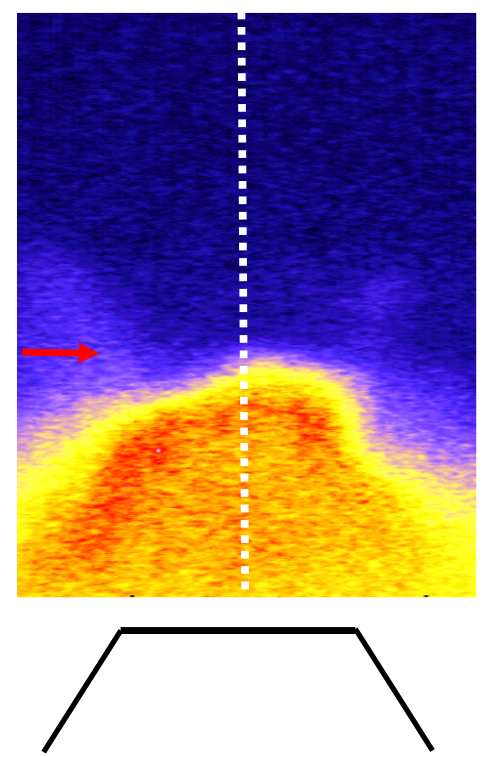

(c)

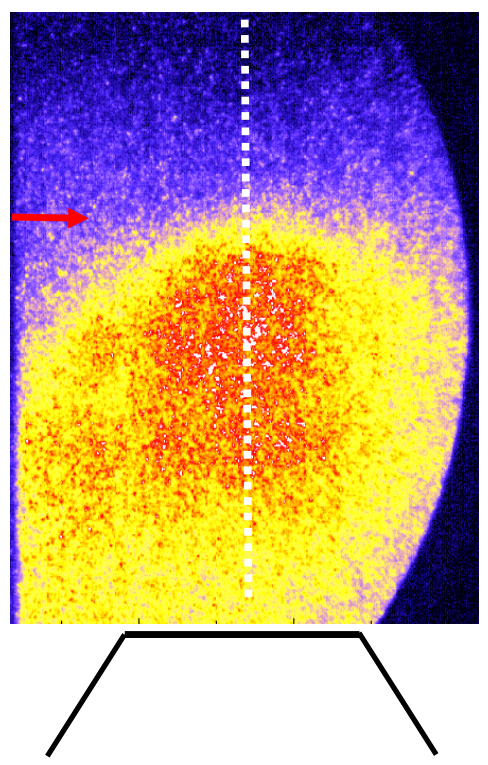

Fig. 4 Raw streaked image with self-emission measurements for (a) short cone target with normal beam irradiation, (b) long cone target with normal beam irradiation, and (c) short cone target with doughnut beam irradiation. Red arrow and dotted line are the maximum compression timing and position of tip center, respectively. Also shown below the images are position of the cone.

$450 \pm 10 \mathrm{~nm}$ with a bandpass filter, where the self-emission intensity is a function of temperature.

Another path was for reflectivity measurements using a velocity interferometer system for any reflector (VISAR) [11]. The spatial and temporal resolutions for the VISAR were approximately $60 \mu \mathrm{m}$ and $140 \mathrm{ps}$, respectively. The velocity per fringe of the interferometer was $6.4 \mathrm{~km} / \mathrm{s} /$ fringe. A YAG laser $(\lambda: 532 \mathrm{~nm}, 10 \mathrm{~ns})$ was employed as a probe beam for VISAR measurements.

The baseline of the implosion dynamics for the conein-shell target was also measured via an x-ray streak camera and through $\mathrm{x}$-ray framing. From the $\mathrm{x}$-ray streak camera data, the implosion velocity and maximum compression timing for each data shot was obtained to make correlations between the timing of observations and optical diagnostics.

The experimental conditions for each shot were (a) the standard "short" cone target irradiated by beams without masks, (b) the "long" cone target irradiated by beams without masks, and (c) the standard "short" cone target irradiated by "doughnut" beams. The three distinct experimental conditions were employed to verify the presence of the preformed plasma and its suppression within two separate schemes.

\section{Experimental Results}

\subsection{Measurements of self-emission at the tip}

Figure 4 shows raw streaked images with the SOP for three different conditions. The "standard" shot (a) is with short $(1 \mathrm{~mm})$ cone-in-shell targets irradiated by nine drive beams without the beam mask. The maximum compression timings for each data point are indicated in the raw data, with each being separately measured by the x-ray streak camera. The figure also shows shots with the long $(5.5 \mathrm{~mm})$ cone (b) irradiated by nine drive beams without the beam mask, and finally, with the short cone target irradiated by the doughnut beam (c).

From the raw data, it is found that the peak of selfemission is around the maximum compression timing for each shot. Moreover, it was observed that early emissions were prevalent in every shot. The early emissions for the (a) short cone and the (c) doughnut beam are from both the tip and side wall, whereas the early emission for the (b) long cone is from the side wall of the cone and moving to the tip. This emission from the wall is likely due to heating by the imploding shell outside the wall. On the other hand, no clear emission due to the imploding shell is observed in other datasets, which may consequently be attributable to preheating being initiated by other potentially dominating sources.

To compare effects of preformed plasma generation, plots of self-emission histories were constructed at the center of the tip for the three data shots. Figure 5 depicts the plots for the three subject data shots. In the plots, $t=0$ is the peak of the Gaussian pulse shape for the implosion drive beam. The temporal evolution suggests that the short cone data (a) shows significant preheating from a very early juncture of laser irradiation $(t \sim-1.0 \mathrm{~ns})$. The plot for the short cone with the doughnut beam (c) also shows some preheating at around $t \sim-0.5 \mathrm{~ns}$. In contrast, there is no evidence of preheating associated with the long 


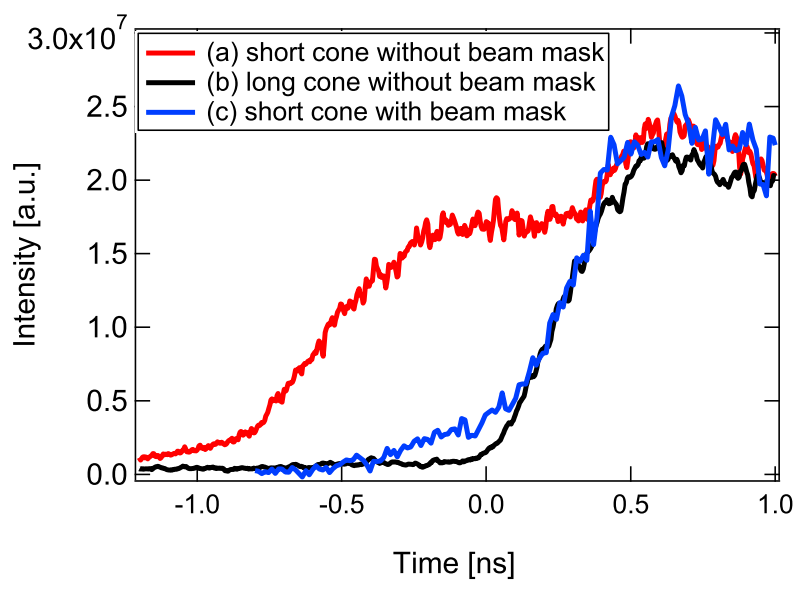

Fig. 5 Temporal profiles of self-emission at around the center of the cone tip for the three experimental conditions shown in Fig. 4.

(a)

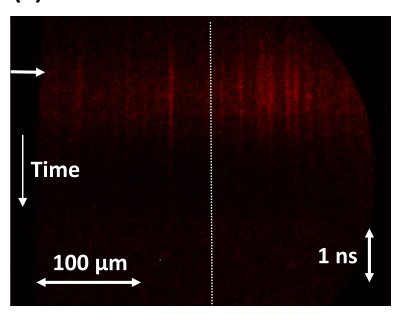

(b)

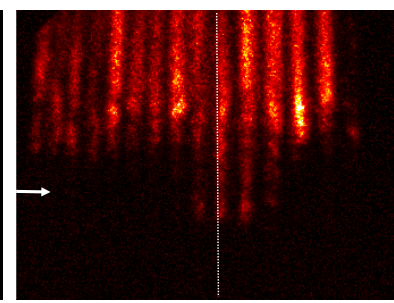

Fig. 6 Raw streaked VISAR images for (a) short cone target without the beam mask and (b) long cone target without the beam mask.

cone data (b). These datasets clearly indicate that preheating due to unconverted light is eliminated by the long cone target.

The influence on the implosion dynamics of the CD shell for the doughnut beam is noteworthy because laser energy and irradiation uniformity were reduced by the mask. The laser energy reduction for the doughnut beam was approximately $16 \%$. Potential issues arising from the implosion dynamics and maximum compression timing were evaluated via the $\mathrm{x}$-ray streak camera and $\mathrm{x}$-ray framing camera data. The measured results suggest that there is no clear difference in these parameters between the normal and doughnut beams.

\subsection{Measurements of reflectivity at the cone tip}

Figure 6 shows raw streaked images with the VISAR for the short cone (a) and long cone (b) targets, which are the same shot data as in the previous section. In typical VISAR measurements, velocity data at a reflecting surface are extracted along with associated fringe shifts. However, in this measurement, the fringe rapidly vanished when the cone tip surface was heated beyond the ionizing temper-

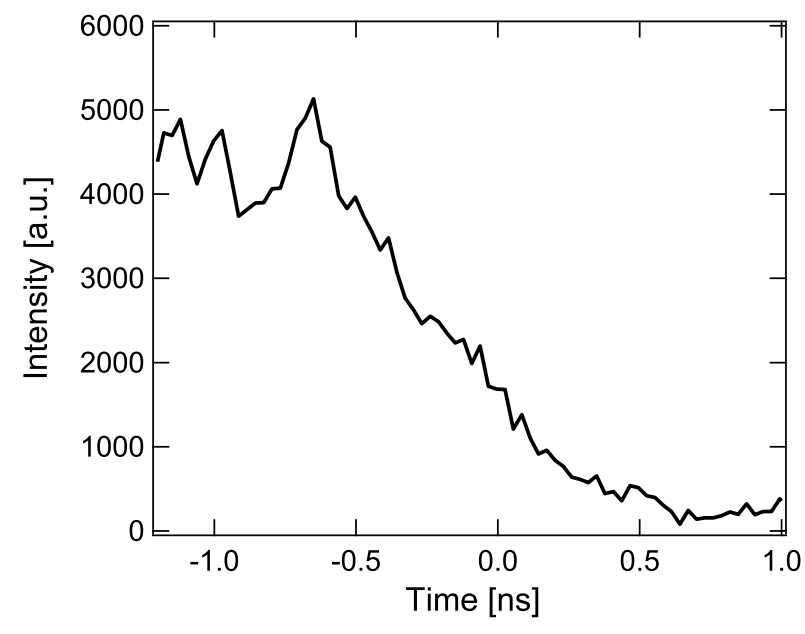

Fig. 7 Temporal profiles of reflectivity at around the center of the cone tip for long cone target without beam mask.

ature, which was observed in previous experiments [12]. Ultimately, as per the VISAR, an estimate of preformed plasma generation levels was rendered with the intensity of reflected light at the central portion of the cone tip.

For the data with the short cone target (a), no clear fringe images were noted for the entire observation period. This implies that the reflectivity might vanish in a very early time segment $(<-0.5 \mathrm{~ns})$ because of plasma generation inside the cone for the short cone target. In contrast, a clear fringe image in early timing was observed for the long cone target (b). A temporal profile of intensities around the center of the tip for the long cone target is shown in Fig. 7. The history of reflectivity shows a constant intensity at the early timing segment and then starts to notably decrease at around $t=-0.5 \mathrm{~ns}$. The reflectivity similarly decreases and subsequently disappears at around $t=0.5 \mathrm{~ns}$. From the self-emission data for the long cone target, self-emission appears at about $t=0 \mathrm{~ns}$, as described in the previous section. The reflectivity drops a little earlier than the rise of self-emission. This might occur because of the reflectivity starting to vanish at gold's melting point temperature, whereby such a temperature is under the detection threshold of the SOP. Thus, the reflectivity data ultimately show a reasonable correlation with the self-emission data.

\subsection{Discussions}

As described in previous sections, the measured data via three separate experimental conditions and two different diagnostic techniques suggest that the long cone target is very effective at suppressing preheating inside a cone. This fact implies that the unconverted light of the implosion laser is a significant source of preformed plasma generation.

In contrast, a short cone with doughnut beams (c) indicates a small degree of preheating inside the cone, sug- 
gesting that there may be another preheating source characteristic of this experimental condition. One probable interpretation is due to the coronal plasma from the shell during the implosion. The shell of the cone-in-shell target is imploded, whereas the subject coronal plasma expands outwardly. The tail of the coronal plasma moves approximately at the speed of sound and finally rolls into a gold cone because the pressure inside the cone is very low (in essence, a vacuum). In this situation, preheating might start at the time that is determined by the velocity of the coronal plasma's tail.

A calculation with the ILESTA-1D simulation suggests that the coronal plasma expands with time, outwardly toward the shell, reaching the edge of the "short" cone entrance at approximately $-0.8 \mathrm{~ns}$ (not shown). As noted above in SOP measurements, self-emission arises at approximately $-0.5 \mathrm{~ns}$, showing good agreement with the subject interpretation. However, note that the coronal plasma does not reach the edge of the long cone entrance during irradiation. This fact suggests that the long cone is very effective at suppressing preheating not only from unconverted light but also from the ablation coronal plasma.

\section{Conclusion}

We have investigated preformed plasma generation inside cones in cone-in-shell targets for fast ignition schemes. We employed self-emission and reflectivity measurements to verify the source(s) of the preformed plasma and to explore the suppression of such plasma. The experimental results show that unconverted light irradiated inside the cone is the major source of the subject preformed plasma generation. It was also concluded that the ablation plasma from the shell is similarly not a negligible source of preformed plasma. These facts suggest that preformed plasma generated by unconverted light and ablation plasma is eliminated using a long cone $(>5.5 \mathrm{~mm}$ ) for cone-inshell targets.

\section{Acknowledgments}

The authors would like to acknowledge the dedicated technical support of the GEKKO-XII facility staff for laser operations, target fabrications, and plasma diagnostics. This work was performed with the support and under the auspices of the NIFS Collaboration Research program. This work was partly supported by the Japan Society for Promotion of Science, KAKENHI Grant Numbers 23340175 and 26630472.
[1] M. Tabak, J. Hammer, M.E. Glinsky, W.L. Kruer, S.C. Wilks, J. Woodworth, E.M. Campbell, M.D. Perry and R.J. Mason, Phys. Plasmas 1, 1626 (1994).

[2] R. Kodama, P.A. Norreys, K. Mima, A.E. Dangor, R.G. Evans, H. Fujita, Y. Kitagawa, K. Krushelnick, T. Miyakoshi, N. Miyanaga, T. Norimatsu, S.J. Rose, T. Shozaki, K. Shigemori, A. Sunahara, M. Tampo, K.A. Tanaka, Y. Toyama, T. Yamanaka and M. Zepf, Nature 412, 798 (2001).

[3] H. Azechi, K. Mima, S. Shiraga, S. Fujioka, H. Nagatomo, T. Johzaki, T. Jitsuno, M. Key, R. Kodama, M. Koga, K. Kondo, J. Kawanaka, N. Miyanaga, M. Murakami, K. Nagai, M. Nakai, H. Nakamura, T. Nakamura, T. Nakazato, Y. Nakao, K. Nishihara, H. Nishimura, T. Norimatsu, P. Norreys, T. Ozaki, J. Pasley, H. Sakagami, Y. Sakawa, N. Sarukura, K. Shigemori, T. Shimizu, A. Sunahara, T. Taguchi, K. Tanaka, K. Tsubakimoto, Y. Fujimoto, H. Homma and A. Iwamoto, Nucl. Fusion 53, 104021 (2013).

[4] A.J. Kemp, F. Fiuza, A. Debayle, T. Johzaki, W.B. Mori, P.K. Patel, Y. Sentoku and L.O. Silva, Nucl. Fusion 54, 5 (2014).

[5] K. Shigemori, Y. Hironaka, H. Nagatomo, S. Fujioka, A. Sunahara, T. Kadono, H. Azechi and K. Shimizu, Appl. Phys. Lett. 102, 183501 (2013).

[6] C. Yamanaka, Y. Kato, Y. Izawa, K. Yoshida, T. Yamanaka, T. Sasaki, M. Nakatsuka, T. Mochizuki, J. Kuroda and S. Nakai, IEEE J. Quantum Electron. 17, 1639 (1981).

[7] F. Girard, L. Suter, O. Landen, D. Munro, S. Regan and J. Kline, Rev. Sci. Instrum. 80, 063104 (2009).

[8] W. Theobald, A.A. Solodov, C. Stoeckl, K.S. Anderson, R. Betti, T.R. Boehly, R.S. Craxton, J.A. Delettrez, C. Dorrer, J.A. Frenje, V.Y. Glebov, H. Habara, K.A. Tanaka, J.P. Knauer, R. Lauck, F.J. Marshall, K.L. Marshall, D.D. Meyerhofer, P.M. Nilson, P.K. Patel, H. Chen, T.C. Sangster, W. Seka, N. Sinenian, T. Ma, F.N. Beg, E. Giraldez and R.B. Stephens, Phys. Plasmas 18, 056305 (2011).

[9] H. Takabe, M. Yamanaka, K. Mima, C. Yamanaka, H. Azechi, N. Miyanaga, M. Nakatsuka, T. Jitsuno, T. Norimatsu, M. Takagi, H. Nishimura, M. Nakai, T. Yabe, T. Sasaki, K. Yoshida, K. Nishihara, Y. Kato, Y. Izawa, T. Yamanaka and S. Nakai, Phys. Fluids 31, 2884 (1988).

[10] J.E. Miller, T.R. Boehly, A. Melchior, D.D. Meyerhofer, P.M. Celliers, J.H. Eggert, D.G. Hicks, C.M. Sorce, J.A. Oertel and P.M. Emmel, Rev. Sci. Instrum. 78, 034903 (2007).

[11] L.M. Barker and R.E. Hollenbach, J. Appl. Phys. 43, 4669 (1972).

[12] K. Otani, K. Shigemori, T. Kadono, Y. Hironaka, M. Nakai, H. Shiraga, H. Azechi, K. Mima, N. Ozaki, T. Kimura, K. Miyanishi, R. Kodama, T. Sakaiya and A. Sunahara, Phys. Plasmas 17, 032702 (2010). 